\title{
Le bissap (Hibiscus sabdariffa L.) : composition et principales utilisations
}

\author{
Mady CISSE ${ }^{1,2}$, Manuel DornieR ${ }^{1 *}$, Mama SAKHO ${ }^{2}$, Augustin NdIAYE $^{3}$, Max REYNES ${ }^{1}$, Oumar SOCK ${ }^{2}$
}

1 UMR 95 Qualisud, Montpellier SupAgro, CIRAD, Montpellier Cedex 5, France dornier@cirad.fr

2 ESP, Dép. GCBA, 5085 Dakar Fann, Sénégal

3 ITA, Route des Pères

Maristes, Dakar Hann, Sénégal TA B-95/16, F-34398

\section{The bissap (Hibiscus sabdariffa L.): composition and principal uses.}

Abstract - Introduction. Hibiscus sabdariffa L. is a herbaceous plant, cultivated largely in tropical and subtropical areas of both hemispheres. This plant is used for its fibre; mainly for its calyx, which is of three types: green, red and dark red. Composition. The red calyxes are the most used and are characterised by their concentration of anthocyanin, which can reach $1.5 \mathrm{~g} \cdot \mathrm{kg}^{-1}$ of dry matter. Delphinidin 3-sambubioside and cyanidin 3-sambubioside are the major anthocyanins with, respectively, (71 and 29)\% of total anthocyanins. Organic acids, minerals and amino acids are present in the calyx, leaves and seeds of $H$. sabdariffa at variable levels depending on the variety and geographical area. Uses. The uses of different parts of $H$. sabdariffa are many and varied both in food and in traditional medicine. The calyx, with high concentration of acids, vitamin $\mathrm{C}$ and especially anthocyanins, is the most used part of the plant. It is mainly used for the production of soft drinks and tonic without alcohol. The seeds of $H$. sabdariffa, with their richness in protein (26\%), fat (20\%) and total sugars (40\%), are widely used in the diet in many African countries. The leaves are a good source of nutrients and are used in Africa in the preparation of sauces.

\section{Senegal / Hibiscus sabdariffa / calyx / leaves / seeds / anthocyanins / proximate composition / uses}

\section{Le bissap (Hibiscus sabdariffa L.) : composition et principales utilisations.}

Résumé - Introduction. Hibiscus sabdariffa L. est une plante herbacée, vivace, localisée dans les zones tropicales et subtropicales des deux hémisphères. L'espèce est exploitée pour sa fibre et principalement pour ses calices qui sont de trois types : vert, rouge et rouge foncé. Composition. Les calices de type rouge sont les plus utilisés et se caractérisent par leur concentration élevée en anthocyanes qui peut atteindre $1,5 \mathrm{~g} \cdot \mathrm{kg}^{-1}$. La delphinidine 3 -sambubioside et la cyanidine 3-sambubioside sont les anthocyanes majoritaires avec, respectivement,

* Correspondance et tirés à part 71 et 29$) \%$ des anthocyanes totaux. Les acides organiques, minéraux et acides aminés sont présents dans les calices, feuilles et graines d'H. sabdariffa à des teneurs variables suivant la variété et la zone géographique. Utilisations. Les utilisations des différentes parties de l'H. sabdariffa sont nombreuses et variées aussi bien dans l'alimentation que dans la médecine traditionnelle. Les calices, du fait de leur concentration élevée en acides, vitamine $\mathrm{C}$ et surtout en anthocyanes, constituent la partie de la plante la plus valorisée. Ils sont surtout utilisés pour la production de boissons désaltérantes et tonifiantes sans alcool. Les graines d'H. sabdariffa de par leur richesse en protéine (26\%), lipides (20 \%) et sucres totaux (40\%) sont très utilisées dans l'alimentation dans de nombreux pays africains. Les feuilles constituent une bonne source de nutriments et sont utilisées en Afrique pour la préparation de sauces.

Sénégal / Hibiscus sabdariffa / calice / feuille / graine / anthocyane / composition globale / utilisation
Fruits, 2009, vol. 64, p. 179-193 (C) 2009 Cirad/EDP Sciences All rights reserved DOI: $10.1051 /$ fruits/2009013 www.fruits-journal.org

RESUMEN ESPAÑOL, p. 193 


\section{Introduction}

Plus de 500 espèces d'Hibiscus sont connues dans le monde. Ces plantes se rencontrent à la fois dans les régions tropicales et subtropicales. Elles possèdent des calices de couleur verte ou rouge. La majorité des variétés sont utilisées comme plantes ornementales à l'exception du type sabdariffa dont deux variétés ont été identifiées [1,2]. Il s'agit d'Hibiscus sabdariffa variété altissima et d'Hibiscus sabdariffa variété sabdariffa $\mathrm{L}$.

Hibiscus sabdariffa L. est une plante de grande taille, vigoureuse, peu ramifiée et très fibreuse [3]. Elle est d'ailleurs cultivée principalement pour ses fibres. La variété sabdariffa L. est une plante touffue pourvue de calices comestibles. Les investigations qui suivent portent essentiellement sur cette dernière variété (figure 1).

Du fait de la crise du secteur agricole, la culture de $H$. sabdariffa est devenue pour certaines localités du Sénégal, en particulier pour le bassin arachidier de la zone centre, une possibilité de diversification de la monoculture de l'arachide [4]. Notre étude bibliographique propose une sélection et une compilation des informations disponibles dans la littérature sur la composition et les utilisations de la plante. Il s'agit d'une synthèse générale destinée à mieux appréhender le potentiel de cette production.

\section{Composition}

\subsection{Calices}

Les calices d' $H$. sabdariffa, principale partie comestible de la plante, ont une composition très variable (tableau I) [1, 2, 5-7].

Excepté pour les teneurs en eau et en lipides, les écarts entre les valeurs minimales et maximales des différents éléments considérés sont importants. Cette variabilité peut être due à plusieurs facteurs tels les conditions de culture, la nature des sols, la pluviométrie et le pays d'origine des calices [1, 2]. La variété est aussi un élément majeur des différences de composition observées [7].
Les calices d' $H$. sabdariffa sont riches en acides organiques. Les acides succinique, oxalique, tartrique et malique sont présents à des concentrations respectives de $[(0,51$, $0,43,0,17$ et 0,12$\left.) \mathrm{g} \cdot 100 \mathrm{~g}^{-1}\right][6,9]$. Les acides succiniques et oxaliques constituent les deux acides organiques majoritaires d'H. sabdariffa. À eux deux, ils représentent $76 \%$ des acides organiques totaux. La teneur en acide ascorbique est très variable même au sein d'espèces cultivées dans la même zone géographique comme cela a été montré au Nigéria [7]. Les sucres présents dans les calices d'H. sabdariffa sont constitués de glucose, fructose et saccharose. Le glucose, avec près de $40 \%$ des sucres totaux, est le sucre majoritaire [6].

L'étude de la composition en minéraux de calices d'H. sabdariffa mise en parallèle avec les concentrations maximales autorisées dans l'alimentation humaine révèle une forte variabilité, fonction de la zone géographique de production (tableau II) [7, 1013]. Dans ces calices, on constate la présence d'oligoéléments, tels que le chrome et le cuivre, alors que d'autres éléments n'ont été mis en évidence que dans certaines origines; c'est le cas, par exemple, du plomb et du nickel détectés dans les seuls calices des cultures du Mali.

La variété influence également la composition minérale [6]. Ainsi des différences significatives ont été observées pour les teneurs en fer, sodium, et potassium selon les variétés rouge et rouge foncée d'H. sabdariffa. Globalement, il apparaît que les calices d'H. sabdariffa constituent une bonne source d'éléments essentiels ( $\mathrm{Ca}, \mathrm{Cu}$, $\mathrm{Fe}, \mathrm{K}, \mathrm{Mn}, \mathrm{Zn}$ ). À l'exception de la teneur en plomb, toutes les valeurs rapportées sont au-dessous du seuil admissible défini par l'organisation mondiale de la santé [10-12] ; elles ne peuvent donc pas constituer un risque sanitaire pour les consommateurs d'H. sabdariffa. Cependant, la teneur en éléments minéraux des plantes dépend de nombreux facteurs parmi lesquels on peut citer les conditions géo-climatiques environnantes, les activités humaines à proximité du champ et les pratiques culturales utilisées. Pour s'assurer de la qualité de la matière végétale, il y aurait lieu d'effectuer une analyse plus systématique de la composition 
des calices en ces éléments minéraux qui, pour certains, sont des métaux lourds.

La présence de $\beta$-carotène et de lycopène à des concentrations respectives de $1,9 \mathrm{mg} \cdot 100 \mathrm{~g}^{-1}$ et $164,3 \mu \mathrm{g} \cdot 100 \mathrm{~g}^{-1}$ de matière fraîche a été signalée dans des calices d'H. sabdariffa [6]. Ces calices contiendraient également des mucilages et des pectines [14-16], ainsi que tous les acides aminés essentiels (tableau III) [2, 17, 18]. Plus de 37 composés volatils y ont été également identifiés parmi lesquels le 1-hexanol, le nonanal, le limonène, le linalool à des concentrations respectives de $(21,9,3,9,2,3$, $1,0) \mathrm{mg} \cdot \mathrm{kg}^{-1}$ de matière sèche [15].

Une des caractéristiques d'H. sabdariffa est également sa richesse en anthocyanes (calices rouges) dont la teneur peut atteindre $1,5 \mathrm{~g} \cdot \mathrm{kg}^{-1}$ de calices secs, teneur comparable à la mûre et supérieure à la plupart des autres végétaux comestibles (tableau IV) [19]. Deux à quatre anthocyanes ont été identifiés selon les variétés d'H. sabdariffa considérées [20-26]. Il s'agit de la delphinidine 3-sambubioside ou hibiscine, de la cyanidine 3-sambubioside ou gossypicyanine, de la delphinidine 3-glucoside et de la cyanidine 3-glucoside. La delphinidine 3-sambubioside est l'anthocyane majoritaire responsable de la couleur rouge-violette des calices [27-28]. Il représente $70 \%$ de la teneur totale en anthocyanes. Les calices d'H. sabdariffa contiennent également d'autres composés polyphénoliques notamment de l'acide protocatéchique [29, 30].

L'activité antioxydante des anthocyanes confère aux boissons à base d' $H$. sabdariffa des propriétés antioxydantes intéressantes, bien qu'environ 10 fois moins élevées que celles du vin rouge [31]. Les anthocyanes sont responsables à $50 \%$ de l'activité antioxydante totale du produit. Des études ont montré que les deux anthocyanes majeurs d'H. sabdariffa, la delphinidine 3sambubioside et la cyanidine 3-sambubioside, sont rapidement absorbés dans le tractus gastro-intestinal chez le rat, ce qui laisse présager une bonne biodisponibilité [31].

La mesure des activités anti-radicalaires d'extraits d'anthocyanes d'H. sabdariffa obtenus par macération de calices dans un mélange éthanol-acide trifluoroacétique

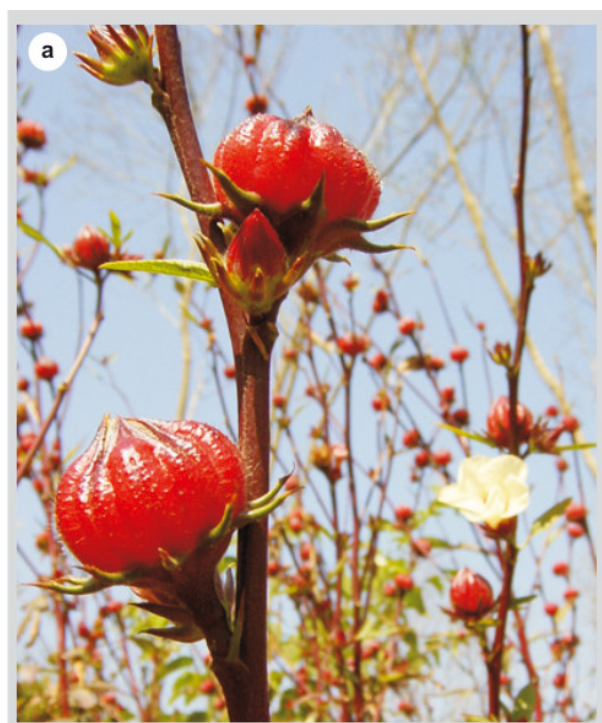

Figure 1.

Hibiscus sabdariffa, variété sabdariffa : (a) tige, feuilles et calices de la variété Koor ; (b) fleur ; (c) fruit et calice de la variété Koor à 120 jours de culture.
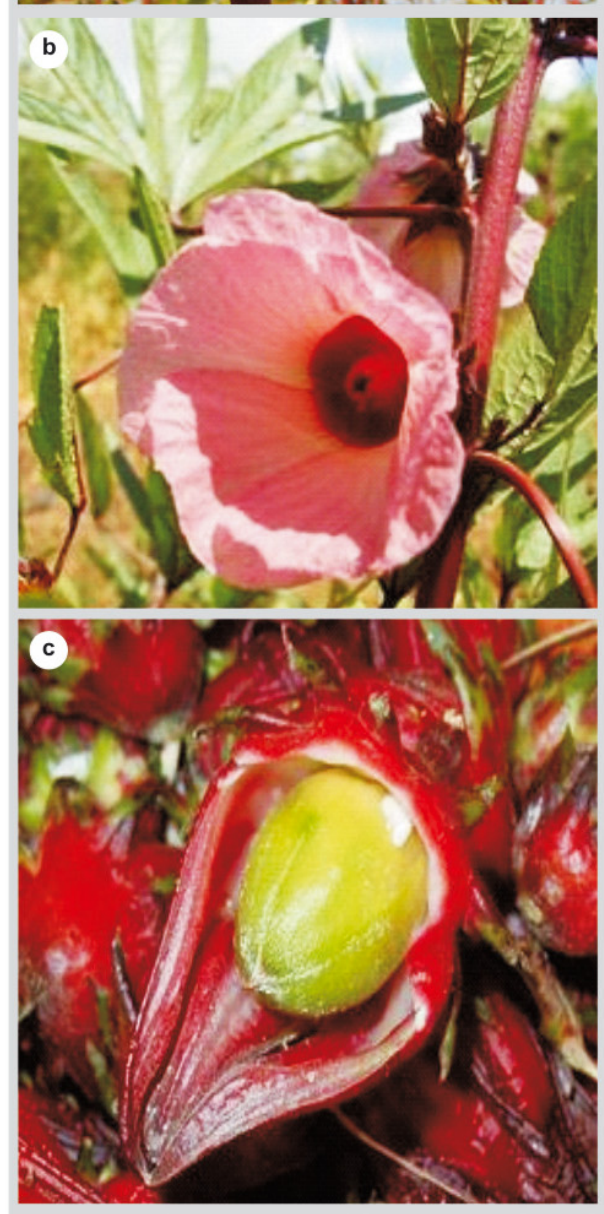

[99 / 1] (vol. / vol.) a révélé des propriétés comparables à celles de l'acide ascorbique [26]. 
Tableau I.

Valeurs minimales, maximales et moyennes des différentes caractéristiques des calices frais d'Hibiscus sabdariffa [1, 2, 5-8].

\begin{tabular}{|c|c|c|c|c|c|c|c|c|c|c|c|c|}
\hline \multirow[t]{2}{*}{$\begin{array}{l}\text { Type de } \\
\text { données }\end{array}$} & Humidité & Protéines & Lipides & Fibres & Cendres & Glucides & $\begin{array}{l}\text { Acide } \\
\text { malique }\end{array}$ & Calcium & Fer & Phosphore & $\begin{array}{c}\text { Acide } \\
\text { ascorbique }\end{array}$ & Anthocyanes \\
\hline & \multicolumn{7}{|c|}{$\left(g \cdot 100 \mathrm{~g}^{-1}\right)$} & \multicolumn{5}{|c|}{$\left(\mathrm{mg} \cdot 100 \mathrm{~g}^{-1}\right)$} \\
\hline Minimales & 84,5 & 0,9 & 0,1 & 2,5 & 4,5 & 3,3 & 0,12 & 1,3 & 2,9 & 40,0 & 6,7 & 150 \\
\hline Moyenne $^{1}$ & $86,3(8)$ & $6,6(8)$ & $2,3(7)$ & $8,8(6)$ & $5,6(5)$ & $8,1(4)$ & 1,36 (3) & $94,0(9)$ & $17,2(9)$ & $191,1(6)$ & $72,0(6)$ & $350(5)$ \\
\hline Maximales & 89,5 & 17,9 & 3,9 & 12,0 & 6,8 & 12,3 & 2,70 & 213,0 & 37,8 & 312,6 & 141,1 & 1500 \\
\hline
\end{tabular}

\section{Tableau II.}

Composition en minéraux des calices d'Hibiscus sabdariffa ( $\mathrm{mg} \cdot 100 \mathrm{~g}^{-1}$ de matière sèche) et teneur maximale autorisée dans l'alimentation humaine [1, 10-13].

\begin{tabular}{|c|c|c|c|c|c|c|c|c|c|c|c|c|}
\hline Origine des calices & Calcium & Cuivre & Chrome & Fer & Magnésium & Manganèse & Nickel & Phosphore & Plomb & Potassium & Sodium & Zinc \\
\hline Nigéria [1] & 1583 & $\begin{array}{l}\text { Non } \\
\text { déterminé }\end{array}$ & - & 37,8 & 316 & $\begin{array}{l}\text { Non } \\
\text { déterminé }\end{array}$ & - & $\begin{array}{l}\text { Non } \\
\text { déterminé }\end{array}$ & - & 2060 & 5,5 & 6,5 \\
\hline Mali [10] & $\begin{array}{c}\text { Non } \\
\text { déterminé }\end{array}$ & 0,6 & 0,6 & 40 & $\begin{array}{c}\text { Non } \\
\text { déterminé }\end{array}$ & 24 & 0,31 & $\begin{array}{c}\text { Non } \\
\text { déterminé }\end{array}$ & 0,18 & $\begin{array}{c}\text { Non } \\
\text { déterminé }\end{array}$ & $\begin{array}{c}\text { Non } \\
\text { déterminé }\end{array}$ & 3,7 \\
\hline $\begin{array}{l}\text { Teneur ou dose } \\
\text { maximale autorisée }\end{array}$ & - & 4 & 12 & $45^{1}$ & - & $2-5^{1}$ & 1 & - & 0 & - & - & 6 \\
\hline
\end{tabular}

Tableau III.

Composition en acides aminés $\left(\mathrm{mg} \cdot \mathrm{g}^{-1}\right.$ de matière sèche) des calices et graines d'Hibiscus sabdariffa.

\begin{tabular}{|c|c|c|c|c|c|c|c|c|c|c|c|c|c|c|c|c|c|c|}
\hline Organe cc & Ala & Arg & Asp & Cys & Gln & Gly & tis & Ile & Leu & Lys & Met & Phe & Pro & Ser & Thr & Trp & Tyr & Val \\
\hline & & & & & & & & & & & & & & & & & & 3,80 \\
\hline żraines $[17,18]$ & (3) & 10,58 & 10,64 & 2,65 & 22,47 & 4,69 & 2,35 & 3,25 & 6,80 & 5,51 & 1,25 & 5,18 & 3,72 & 4,48 & 3,80 & 0,51 & 3,46 & 3,57 \\
\hline
\end{tabular}

Cependant, les anthocyanes d'H. sabdariffa sont réputés pour leur instabilité [19, 32-34]. Ils sont facilement dégradés pendant les traitements thermiques ou durant leur stockage à température ambiante. Après chauffage, la coloration rouge vire progressivement au brun. Cette instabilité, aussi bien dans des solutions aqueuses simples que dans des formulations complexes de produits alimentaires, est le principal facteur limitant de l'utilisation des extraits d'H. $s a b$ dariffa comme colorant. Une plus grande stabilité ouvrirait à cette variété le marché des colorants naturels qui, évalué à 940 M USD, enregistre une croissance annuelle de l'ordre de $5 \%$ comparé à celui des colorants artificiels (400 M USD), qui lui n'augmente que de (2 à 3) \% par an [35].

\subsection{Graines}

À partir d'un échantillon de 1000 graines, il a été mesuré que la graine d'H. sabdariffa avait un diamètre moyen principal de 


\section{Tableau IV.}

Principaux anthocyanes et teneurs trouvés dans certains fruits [19].

\begin{tabular}{|c|c|c|}
\hline Fruits & Anthocyanes & $\begin{array}{c}\text { Teneur } \\
\left(\mathrm{mg} \cdot 100 \mathrm{~g}^{-1}\right)\end{array}$ \\
\hline $\begin{array}{l}\text { Black chokeberry } \\
\text { (Aronia melanocarpa) }\end{array}$ & $\begin{array}{l}\text { Cy 3-galactoside } \\
\text { Cy } 3 \text {-arabinoside } \\
\text { Cy 3-syloside }\end{array}$ & 1050 \\
\hline $\begin{array}{l}\text { Cerise sucrée } \\
\text { (Prunus avium var. Bigareau) }\end{array}$ & $\begin{array}{l}\text { Cy 3-rhamnoglucoside } \\
\text { Cy 3-glucoside } \\
\text { Pn 3-rutinoside }\end{array}$ & $350-450$ \\
\hline $\begin{array}{l}\text { Cerise aigre } \\
\text { (Prunus cerasus L. var. Montmorency) }\end{array}$ & $\begin{array}{c}\text { Pn 3-rutinoside } \\
\text { Cy 3-glucoside } \\
\text { Cy 3-rutinoside } \\
\text { Cy 3-sophoroside } \\
\text { Cy } 3-2^{G} \text { glucosylrutinoside }\end{array}$ & $35-82$ \\
\hline $\begin{array}{l}\text { Prune } \\
\text { (Prunas salicina cv. Sordum). }\end{array}$ & $\begin{array}{l}\text { Cy 3-rhamnoglucoside } \\
\text { Cy 3-glucoside }\end{array}$ & 30 \\
\hline $\begin{array}{l}\text { Myrtille } \\
\text { (Vaccinium corymbosum L.) }\end{array}$ & $\begin{array}{l}\text { Dp 3-galactoside } \\
\text { Dp 3-arabinoside } \\
\text { Mv 3-galactoside } \\
\text { Mv-arabinoside }\end{array}$ & $25-495$ \\
\hline $\begin{array}{l}\text { Canneberge } \\
\text { (Vaccinium macrocarpon Ait.) }\end{array}$ & $\begin{array}{l}\text { Pn 3-galactoside } \\
\text { Pn 3-arabinoside } \\
\text { Cy 3-galactoside } \\
\text { Cy 3-arabinoside }\end{array}$ & 78 \\
\hline $\begin{array}{l}\text { Framboise noire } \\
\text { (Rubus spp. var Cumberland) }\end{array}$ & $\begin{array}{c}\text { Cy 3-glucoside } \\
\text { Cy 3-rutinoside } \\
\text { Cy 3-sambubioside } \\
\text { Cy 3-xylosylrutinoside }\end{array}$ & 428 \\
\hline $\begin{array}{l}\text { Fraise } \\
\text { (Fragaria spp.) }\end{array}$ & $\begin{array}{l}\text { Pg 3-glucoside } \\
\text { Cy 3-glucoside }\end{array}$ & $450-700$ \\
\hline $\begin{array}{l}\text { Raisin } \\
\text { (Vitis vinifera L.) }\end{array}$ & $\begin{array}{c}\text { Mv 3-monoglucoside } \\
\text { Pn 3-monoglucoside } \\
\text { Dp 3-monoglucoside } \\
\text { Mv 3-monoglucoside-p-coumarate } \\
\text { Pn 3-monoglucoside-p-coumarate }\end{array}$ & $30-750$ \\
\hline Hibiscus sabdariffa & $\begin{array}{l}\text { Dp 3-sambubioside } \\
\text { Cy 3-sambubioside } \\
\text { Dp 3-glucoside } \\
\text { Cy 3-glucoside }\end{array}$ & 150 \\
\hline $\begin{array}{l}\text { Mûre } \\
\text { (Rubus fruticosus L.) }\end{array}$ & $\begin{array}{l}\text { Cy 3-glucoside } \\
\text { Cy 3-rutinoside }\end{array}$ & $67-230$ \\
\hline $\begin{array}{l}\text { Orange sanguine } \\
\text { (Citrus sinensis L.) }\end{array}$ & $\begin{array}{c}\text { Cy 3-glucoside } \\
\text { Cy 3-(4"-acetyl)-glucoside }\end{array}$ & $70-100$ \\
\hline
\end{tabular}




\section{Tableau V.}

Composition des graines d'Hibiscus sabdariffa $\left(\mathrm{g} \cdot 100 \mathrm{~g}^{-1}\right)[2,17,18]$.

\begin{tabular}{lcccccc} 
Type de données & Humidité & Protéines & Lipides & Fibres & Cendres & Glucides \\
Minimum & 5,6 & 19,6 & 16 & 1,2 & 4,8 & 23,8 \\
Moyenne $^{1}$ & $9,3(8)$ & $26,2(8)$ & $20,2(8)$ & $9,0(7)$ & $5,9(7)$ & $40,4(7)$ \\
Maximum & 12,9 & 31,0 & 23,3 & 16,8 & 7,0 & 51,7 \\
& \multicolumn{7}{l}{${ }^{1}$ Entre parenthèses : le nombre de valeurs pris en compte dans le calcul de la moyenne. }
\end{tabular}

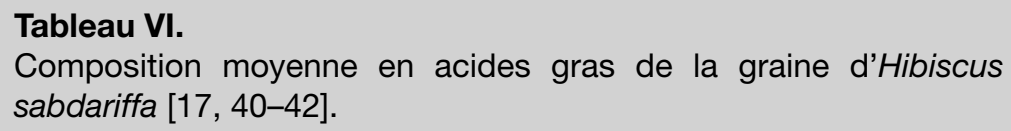

Tableau VI.

Composition moyenne en acides gras de la graine d'Hibiscus sabdariffa [17, 40-42].

$\begin{array}{lcc}\text { Acide gras } & & \text { \% des lipides } \text { totaux }^{1} \\ \text { C14:0 } & \text { Myristique } & 0,31 \\ \text { C16:0 } & \text { Palmitique } & 21,65 \\ \text { C16:1 } & \text { Palmitoléique } & 0,44 \\ \text { C18:0 } & \text { Stéarique } & 5,47 \\ \text { C18:1 } & \text { Oléique } & 30,90 \\ \text { C18:2 } & \text { Linoléique } & 39,16 \\ \text { C18:3 } & \gamma \text {-linolénique } & 0,57 \\ \text { C20:0 } & \text { Arachidique } & 0,72 \\ \text { C20:1 } & \text { Eicosénoïque } & 0,08 \\ \text { C20:3 } & \text { Eicosatriénoïque } & 0,34 \\ \text { C22:0 } & \text { Behénique } & 0,37 \\ 1 \text { Moyenne des valeurs données par les références. } & \end{array}$

$5,6 \mathrm{~mm}$, un diamètre intermédiaire de $5,2 \mathrm{~mm}$ et minimal de $2,8 \mathrm{~mm}$. Le poids de ces 1000 graines est de 35,6 g [36].

La chaleur spécifique de la graine à $80{ }^{\circ} \mathrm{C}$ est de $2,97 \mathrm{~kJ} \cdot \mathrm{kg}^{-1} \cdot \mathrm{K}^{-1}$. Cette valeur est comprise dans l'intervalle de $(2,1$ et $5,3) \mathrm{kJ} \cdot \mathrm{kg}^{-1} \cdot \mathrm{K}^{-1}$ rapportés pour différentes graines oléagineuses [37]. La connaissance de la chaleur spécifique est utile lors du traitement thermique des graines, afin de pouvoir prévoir son comportement à la chaleur.

Les éléments trouvés dans les calices d'H. sabdariffa se retrouvent également dans les graines, cependant leurs teneurs sont généralement plus importantes dans les graines que dans les calices (tableau $V$ ) $[2,17,18]$.
Les graines d'H. sabdariffa présentent des concentrations très importantes en protéines (26\%), lipides (20\%) et sucres totaux (40\%). Les glucides sont constitués de saccharose, glucose et amidon à des teneurs moyenne respectives de $(17,6,4,0$ et 16,1) $\mathrm{g} \cdot 100 \mathrm{~g}^{-1}$ de graines entières fraîches. Ce sont donc de bonnes sources de protéines et de lipides.

L'acide glutamique (22\%), la glycine (18\%) et l'acide aspartique (11\%) sont les acides aminés majoritaires dans les graines (tableau III) [17, 18]. Tous les acides aminés essentiels sont présents, cependant, dans ce groupe, la leucine (7\%), la phénylalanine $(5 \%)$ et la thréonine (4\%) sont les plus représentées.

La concentration totale en acides aminés essentiels de la référence protéique de la FAO [17] est de $36 \mathrm{~g} \cdot 100 \mathrm{~g}^{-1}$ de protéine, alors qu'elle est de $39,5 \mathrm{~g} \cdot 100 \mathrm{~g}^{-1}$ de protéine pour les graines d'H. sabdariffa. Selon le modèle de référence de la FAO, les acides aminés limitants seraient la valine, l'isoleucine, et le tryptophane, tandis que tous les acides aminés soufrés ne le seraient pas. La teneur en lysine des graines est identique à celle de la protéine de référence de la FAO. Il serait donc envisageable d'utiliser les graines d'H. sabdariffa pour enrichir en lysine des aliments qui en sont déficients.

Avec une teneur moyenne de $20 \%$, la graine d'H. sabdariffa présente une richesse en huile proche de celle d'autres graines comme celles de la tomate [38] ou des fruits du baobab [39]. L'huile brute des graines est un liquide de couleur jaune verdâtre à température ambiante qui se compose à plus de $70 \%$ d'acides gras insaturés (tableau VI) [17, 40-42]. L'acide linoléique 


\section{Tableau VII.}

Principales caractéristiques physico-chimiques de l'huile brute des graines d'Hibiscus sabdariffa [41, 42].

\begin{tabular}{|c|c|c|c|c|c|c|c|c|}
\hline Références & $\begin{array}{l}\text { Acidité } \\
\text { (\% acide } \\
\text { oléique) }\end{array}$ & $\begin{array}{l}\text { Indice de } \\
\text { peroxyde } \\
\left(\mathrm{mEq} \cdot \mathrm{kg}^{-1}\right)\end{array}$ & $\begin{array}{l}\text { Indice de } \\
\text { réfraction } \\
\text { à } 40^{\circ} \mathrm{C}\end{array}$ & $\begin{array}{l}\text { Densité } \\
\text { à } 25^{\circ} \mathrm{C} \\
\left(\mathrm{kg} \cdot \mathrm{L}^{-1}\right)\end{array}$ & $\begin{array}{l}\text { Viscosité } \\
\text { à } 25^{\circ} \mathrm{C} \\
\text { (cP) }\end{array}$ & $\begin{array}{c}\text { Indice } \\
\text { d'iode } \\
\left(\mathrm{g} \cdot 100 \mathrm{~g}^{-1}\right)\end{array}$ & $\begin{array}{c}\text { Indice de } \\
\text { saponification } \\
\left(\mathrm{mg} \mathrm{KOH} \cdot \mathrm{g}^{-1}\right)\end{array}$ & $\begin{array}{l}\text { Stabilité à } \\
\text { l'oxydation } \\
\text { (h) }\end{array}$ \\
\hline [41] & 2,24 & 8,63 & 1,48 & 0,92 & 15,85 & $\begin{array}{c}\text { Non } \\
\text { déterminé }\end{array}$ & $\begin{array}{c}\text { Non } \\
\text { déterminé }\end{array}$ & 15,53 \\
\hline [42] & $\begin{array}{c}\text { Non } \\
\text { déterminée }\end{array}$ & 11,86 & 1,47 & 0,92 & $\begin{array}{c}\text { Non } \\
\text { déterminée }\end{array}$ & 81,45 & 194,95 & $\begin{array}{c}\text { Non } \\
\text { déterminée }\end{array}$ \\
\hline
\end{tabular}

\begin{tabular}{|c|c|c|c|c|c|c|c|}
\hline $\begin{array}{l}\text { Cultivars d'Hibiscus sabdariffa identifiés à } \\
\text { partir de la couleur des calices }\end{array}$ & Calcium & Magnésium & Manganèse & Potassium & Sodium & Fer & Zinc \\
\hline Rouge clair & 620 & 460 & 4,1 & 1300 & 620 & 9,1 & 5,6 \\
\hline Rouge & 590 & 480 & 3,9 & 1390 & 590 & 9 & 5,5 \\
\hline Rouge foncé & 680 & 420 & 4,0 & 1350 & 680 & 8,8 & 5,9 \\
\hline
\end{tabular}

(C18:2) est le plus abondant (39\%); il est suivi de l'acide oléique (C18:1) à une concentration de $31 \%$. L'acide palmitique (C16:0) est l'acide gras saturé le plus abondant $(21 \%)$; il est suivi de l'acide stéarique (C18:0), avec une teneur de $6 \%$.

Les propriétés physiques et chimiques de l'huile brute révèlent que l'huile a un indice de saponification élevé mais un indice d'iode faible (tableau VII) [41, 42]. Ce dernier indice indique le degré d'insaturation tandis que l'indice de saponification reflète le poids moléculaire moyen. Les valeurs de l'indice de réfraction et de la densité spécifique sont dans la gamme des valeurs observées dans la plupart des huiles végétales brutes [43]. L'indice de peroxyde qui indique l'état d'oxydation de la matière grasse est légèrement élevé pour une huile brute $\left(10 \mathrm{mEq} \cdot \mathrm{kg}^{-1}\right)$. La viscosité de l'huile d'H. sabdariffa est inférieure à celle des huiles de maïs, de soja et d'olive [44, 45].

L'huile présente une valeur de stabilité à l'oxydation très élevée de 15 h qui pourrait être partiellement attribuée à son contenu élevé en tocophérol, de l'ordre de $2 \mathrm{~g} \cdot \mathrm{kg}^{-1}$ [43]. Cette concentration est deux fois plus élevée que celle rencontrée dans l'huile des graines de tomate [38], six fois plus que celle de l'huile d'olive qui contient en moyenne $350 \mathrm{mg} \cdot \mathrm{kg}^{-1}$ de tocophérol [45] et 20 fois plus que celle de l'huile de pépins de raisin [46]. Le tocophérol le plus abondant dans l'huile de graines d'H. sabdariffa est le $\gamma$ tocophérol. Les teneurs en $\alpha$-, $\gamma$-, et $\delta$-tocophérol sont respectivement de $(25,0,74,5$ et $0,5) \%$; ils sont semblables aux teneurs mesurées dans l'huile de maïs raffinée [(17, $78,3) \%$ [41]. Les graines d'H. sabdariffa constituent donc une bonne source d'antioxydants liposolubles.

L'analyse de la composition en minéraux des graines provenant de trois cultivars d'H. sabdariffa révèle que potassium, sodium, magnésium et calcium sont les constituants majoritaires, alors que manganèse, fer et zinc sont présents en faible quantité (tableau VIII) [8]. Le cultivar rouge foncé présente les teneurs les plus élevées en potassium et sodium.

La graine d' $H$. sabdariffa contient plus de 25 composés volatiles [47] dont des hydrocarbures, des alcools, et des aldéhydes $\left(\mathrm{C}_{8}\right.$ à $\left.\mathrm{C}_{13}\right)$. Ces composés sont importants pour l'arôme de l'huile dont la typicité peut être mise à profit en cuisine. Ils peuvent être 
Tableau IX.

Principales caractéristiques des feuilles d'Hibiscus sabdariffa [2, 48].

\begin{tabular}{|c|c|c|c|c|c|c|c|c|c|c|c|c|c|c|c|}
\hline \multirow[t]{2}{*}{ Références } & Eau & Protéines & Lipides & $\begin{array}{c}\text { Glucide } \\
\text { totaux }\end{array}$ & Fibres & Cendres & $\begin{array}{c}\text { Acide } \\
\text { malique }\end{array}$ & Calcium & Phosphore & Fer & -carotène & Thiamine & Riboflavine & Niacine & $\begin{array}{c}\text { Acide } \\
\text { ascorbique }\end{array}$ \\
\hline & \multicolumn{7}{|c|}{$\left(g \cdot 100 \mathrm{~g}^{-1}\right)$} & \multicolumn{8}{|c|}{$\left(\mathrm{mg} \cdot 100 \mathrm{~g}^{-1}\right)$} \\
\hline [2] Philippines & 86,2 & 1,7 & 1,1 & 10,0 & - & 1,0 & 1,3 & 180,0 & 40,0 & 5,4 & - & - & - & - & - \\
\hline Guatemala & 89,2 & 1,1 & 2,6 & - & 12,0 & 6,9 & - & 1,3 & 273,2 & 9,0 & 0,03 & 0,1 & 0,3 & 3,8 & 6,7 \\
\hline [48] & 85,6 & 3,3 & 0,3 & 9,2 & 1,6 & - & - & 213,0 & 93,0 & 4,8 & 4,10 & 0,2 & 0,5 & 1,2 & 54,0 \\
\hline
\end{tabular}

Tableau $X$.

Différentes utilisations alimentaires de l'Hibiscus sabdariffa.

\begin{tabular}{lcc} 
Partie de la plante & Exemples d'utilisation & Références \\
\hline Calice & $\begin{array}{c}\text { Fabrication de glace } \\
\text { Fabrication de gelée, confiture } \\
\text { Préparation de boissons et infusions } \\
\text { Ingrédients dans les aliments } \\
\text { Vin } \\
\text { Vinaigre }\end{array}$ & {$[1,5,8,32,40]$} \\
Feuille & $\begin{array}{c}\text { Sauces aigres } \\
\text { Légumes feuilles }\end{array}$ & {$[53,54]$} \\
Graine & Fabrication de condiments & {$[49]$} \\
& Produit de substitution de la viande & {$[2,51,53]$} \\
& Production de farine & {$[55-57]$} \\
Production d'huile & {$[17,18,41]$}
\end{tabular}

utiles pour l'identification d'huiles provenant de graines de différentes origines géographiques.

\subsection{Feuilles}

La variabilité observée au niveau des calices et des graines d'H. sabdariffa cultivé dans différentes régions se retrouve également pour la composition des feuilles (tableau IX) $[2,48]$. Par exemple, les teneurs en phosphore et fer seraient respectivement de (40 et 5) $\mathrm{mg} \cdot 100 \mathrm{~g}^{-1}$ pour des feuilles de plantes cultivées aux Philippines alors qu'elles seraient respectivement de (273 et 9) $\mathrm{mg} \cdot 100 \mathrm{~g}^{-1}$ pour des feuilles provenant du Guatemala. Ces feuilles constituent une bonne source de nutriments, c'est pourquoi elles entrent dans l'alimentation des populations rurales des pays producteurs [49-51].

\section{Utilisations d'H. sabdariffa}

Toutes les parties des plants d'H. sabdariffa (calice, tige, feuille) sont utilisées soit dans l'alimentation, soit dans la médecine traditionnelle, soit dans l'industrie textile.

\subsection{Utilisations alimentaires}

L'espèce $H$. sabdariffa est utilisé dans l'alimentation humaine et dans l'industrie agroalimentaire. La plante est exploitée pour ses calices, feuilles et graines (tableau X).

\subsubsection{Calices}

Les calices, du fait de leur concentration élevée en acides, pectines, vitamine $C$ et surtout en anthocyanes, constituent la partie de la plante la plus utilisée. Ils interviennent dans la production de boissons désaltérantes et tonifiantes sans alcool.

Ces boissons sont toutes élaborées à partir d'un extrait aqueux obtenu après trempage des calices dans de l'eau chaude ou à température ambiante. Après filtration, du sucre ainsi que d'autres ingrédients, tels que d'autres jus de fruits, des aromatisants, des morceaux de fruits (ananas, fraise, gingembre), etc., peuvent être ajoutés selon le pays pour la confection de la boisson finale. Cette boisson largement répandue en Afrique et en Asie est connue sous plusieurs appellations. Au Sénégal où elle est très appréciée, elle est nommée "bissap" et sa consommation est maximale pendant le mois de ramadan. Au Mali, en Côte d'Ivoire et au Burkina Faso, la boisson est appelée "da bilenni". En Égypte, elle est plus connue sous la dénomination de "boisson des pharaons". Au Soudan l'appellation "thé de karkadé" 


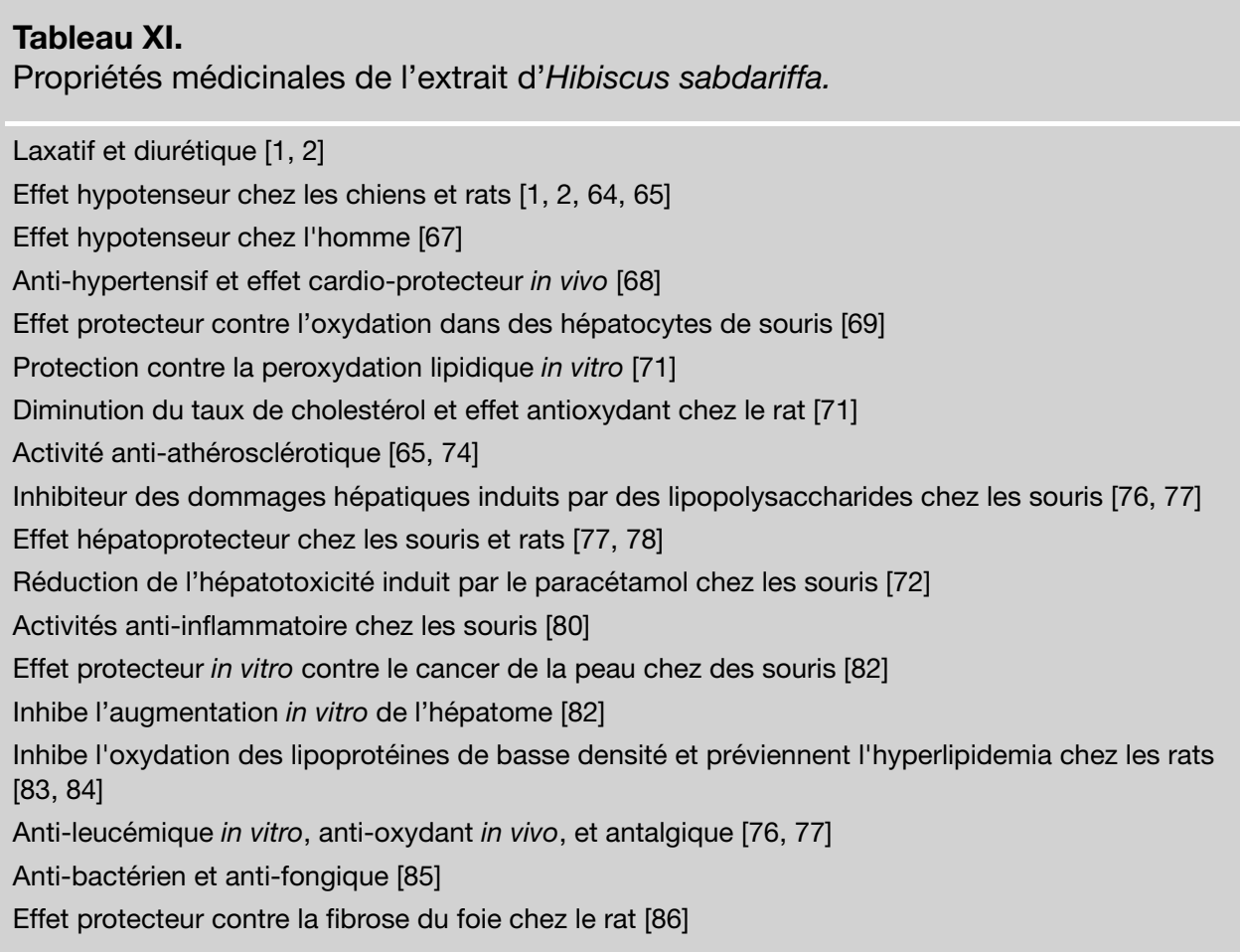

est courante ; cette boisson est alors consommée froide ou chaude selon la saison. En Jamaïque, une boisson traditionnelle de Noël est préparée à partir de calices d'H. sabdariffa avec du gingembre, du sucre et de l'eau bouillante. Le liquide est filtré et servi avec de la glace et souvent avec du rhum. Au Nigéria, la boisson, appelée "zobo ", est tout autant appréciée par toutes les couches sociales de la population. Dans ce pays, sa popularité s'est récemment accrue en raison des vertus médicinales qui lui sont attribuées et de l'augmentation par ailleurs du prix d'autres boissons non alcoolisées importées. En Malaisie, la boisson attire l'attention de beaucoup de fabricants de jus de fruits, tandis qu'elle est traditionnellement très consommée en infusion ou boisson fraîche en Thaillande.

La production de confiture, gelée et dessert à partir des calices est aussi largement répandue. Les confitures d'H. sabdariffa sont appréciées aux États-Unis, en Australie, au Sénégal, dans les Caraibes et en Asie [3]. Les calices sont utilisés également pour fabriquer une boisson fermentée alcoolisée qui s'apparenterait à du vin [52].
Les extraits de calices sous forme de concentré ou de poudre séchée sont utilisés comme colorant naturel dans les industries alimentaires (pâtisserie, jus de fruits, boissons, etc.) et pharmaceutiques. Cependant, actuellement, leur emploi est limité et pose problème du fait de l'instabilité des pigments lors de la conservation du produit [21, $27,36]$.

\subsubsection{Graines}

Les graines d'H. sabdariffa sont riches en protéines, aussi, au Bénin, sont-elles utilisées pour la fabrication de condiments traditionnels par cuisson puis fermentation. Différents produits appelés " iru ", "afitin ", "sonru" ou "yanyanku " sont obtenus en fonction de la durée de fermentation. Des produits similaires sont trouvés dans d'autres régions comme le "dawadawa " au Nigéria et au Ghana [55], le "dadawa basso " et le " dadawa kalwa" au Nigéria [56], le " soumbala" au Burkina Faso [59], le "nététu" au Sénégal [60], le "natto" au Japon et le "kinema" au Népal [57, 61]. Au nord du Nigéria, les graines sont fermentées en présence de quelques épices pour préparer un 


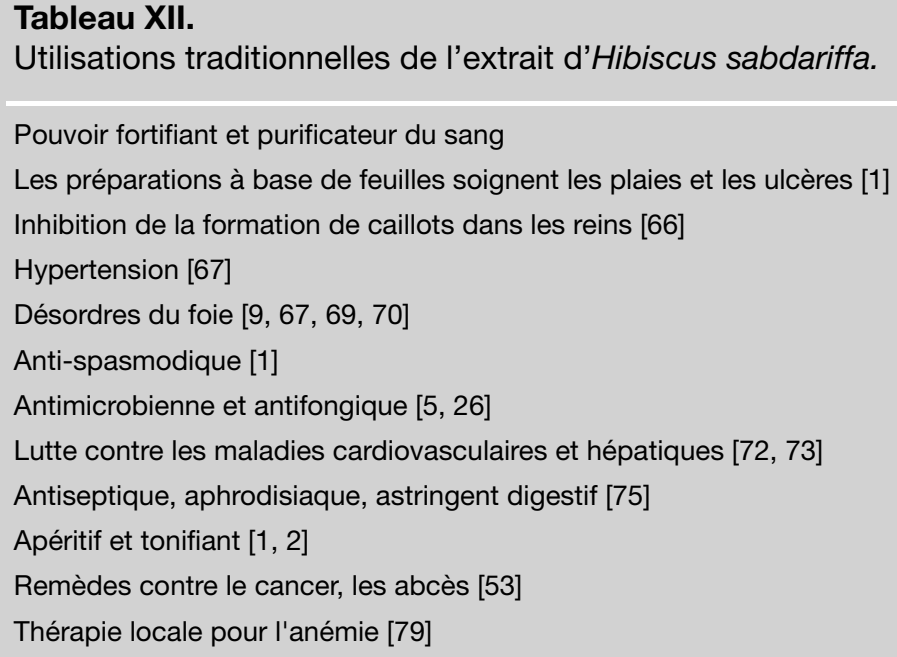

aliment connu sous le nom de "mungza ntusa " [41]. Au Soudan et au Nigéria, elles sont utilisées pour élaborer un produit de remplacement de la viande appelé viande d'oseille, le "furundu ", qui est traditionnellement préparé en faisant fermenter, pendant 9 jours, les graines préalablement cuites [58].

Les graines contiennent environ $20 \%$ de matières grasses. L'huile est constituée essentiellement d'acides gras insaturés. Elle est riche en tocophérols. Son profil acide linoléique/acide oléique est caractéristique [38, 41]. Cette huile est traditionnellement utilisée en cuisine au Tchad, en Tanzanie et en Chine [3]. Elle peut entrer également dans la fabrication de savon et de produits cosmétiques.

Les graines d'H. sabdariffa présentent donc un potentiel intéressant comme sources de protéines et de matières grasses. Une meilleure valorisation de cette partie de la plante pourrait contribuer au développement de sa culture.

\subsubsection{Feuilles}

La composition des feuilles d'H. sabdariffa est également propice à leur utilisation dans l'alimentation humaine. Au Sénégal, ces feuilles sont utilisées pour fabriquer une sauce aigre, épaisse, appelée " bëkëj ", servie avec le riz au poisson. Au Mali, elles sont bouillies pour fabriquer des sauces accom- pagnant différents plats à base de tubercules [3, 35].

En dépit de l'abondance de ces produits sur les marchés locaux, la production de feuilles et de calices d' $H$. sabdariffa pour la consommation domestique en Afrique n'aurait encore jamais été quantifiée [62, 63].

\subsection{Utilisations médicinales}

L'espèce H. sabdariffa aurait de nombreuses propriétés thérapeutiques. Elle est utilisée dans la plupart des médecines traditionnelles aussi bien dans les pays du Sud que dans les pays du Nord. Néanmoins, seul un nombre limité de ces propriétés médicinales a fait l'objet d'études cliniques menées pour la majeure partie sur des animaux (tableau XI).

Plusieurs de ces propriétés médicinales sont attribuées aux concentrations élevées en acides organiques, notamment en acide malique, ascorbique et acide citrique [88]. D'autres activités biologiques seraient liées aux composés anthocyaniques qui sont dotés d'activités antioxydantes importantes [35, 89].

Deux études cliniques, l'une en Iran et l'autre au Mexique [69], réalisées respectivement sur 54 et 75 patients, ont mis en évidence que la consommation journalière d'extrait d'H. sabdariffa diminuerait de manière significative la tension artérielle 
chez les sujets hypertendus. Cependant, en dépit de l'utilisation populaire de cette plante dans le domaine de la pharmacologie (tableau XII), peu ou pas d'informations ont été fournies jusqu'à présent sur sa toxicité. Des travaux complémentaires seraient donc nécessaires dans ce domaine.

\section{Conclusion}

Les feuilles et les graines d'H. sabdariffa, de par leur richesse en protéines, lipides et éléments minéraux et du fait de leur disponibilité, entrent dans l'alimentation des populations rurales des pays en voie de développement. Les calices rouges sont utilisés dans l'alimentation humaine pour la production de confiture, gelée et surtout de boissons rafraichissantes présentant une couleur rouge attractive et un goût plus ou moins acidulé selon les variétés. La couleur rouge est due à la richesse des calices en anthocyanes qui présenteraient plusieurs intérêts nutritionnels et médicinaux. Ces potentialités ont entrainé une augmentation de la demande internationale en calices secs, ce qui constitue une source de revenus importante pour les agriculteurs des pays en voie de développement. Cependant, la relative instabilité des anthocyanes trouvés dans H. sabdariffa limite leur utilisation pour l'élaboration de boissons et leur emploi comme colorant naturel que ce soit dans l'industrie alimentaire ou dans d'autres secteurs tels que les industries pharmaceutique et cosmétique.

Des recherches complémentaires sur les propriétés antioxydantes et thérapeutiques de la plante mériteraient d'être menées. L'étude des différents mécanismes de dégradation des anthocyanes d' $H$. sabdariffa doit être envisagée en tenant compte de plusieurs facteurs tels que la composition des extraits, les températures de traitement, la teneur en oxygène dissous ou l'exposition à la lumière. Enfin, pour obtenir des calices d'H. sabdariffa de qualités physico-chimique et nutritionnelle optimales, il serait essentiel d'améliorer l'organisation des filières de production et de rechercher les meilleures conditions et techniques de culture.

\section{Remerciements}

Ce travail bibliographique s'intègre dans le cadre du projet "Couplage de procédés membranaires pour la production d'extraits anthocyaniques: application à Hibiscus sabdariffa" qui bénéficie d'un support financier du réseau des chercheurs en Génie des Procédés Appliqués à l'Agroalimentaire (GP3A) de l'Agence Universitaire de la Francophonie (AUF).

\section{Références}

[1] Kerharo J., Adam J.G., La pharmacopée sénégalaise traditionnelle - Plantes médicinales et toxiques, Vigot Frères, Paris, France, 1974.

[2] Morton J.F., Roselle, in: Dowling C.F. (Ed)., Fruits of warm climates, Media, Inc., Greensborough, USA, 1987, pp. 281-286.

[3] McClintock N.C., El Tahir I.M., Hibiscus sabdariffa L., in: Grubben G.J.H., Denton, O.A. (Ed.), PROTA 2 (Plant Resources of Tropical Africa): vegetables [CD-Rom], PROTA, Wagening., Neth., 2004.

[4] Cisse M., Dornier M., Sakho M., Mar Diop C., Reynes, M., Sock O., La production de bissap (Hibiscus sabdariffa L.) au Sénégal, Fruits 64 (1) (2009) 1-14.

[5] D'Heureux-Calix F., Badrie N., Consumer acceptance and physicochemical quality of processed red sorrel/roselle (Hibiscus sabdariffa L.) sauces from enzymatic extracted calyces, Food Serv. Technol. 4 (2004) 141148.

[6] Wong P.K., Yusof S., Ghazali H.M., Che Man Y.B., Physicochemical characteristics of roselle (Hibiscus sabdariffa L.), Nutr. Food Sci. 32 (2002) 68-73.

[7] Babalola S.O., Babalola A.O., Aworh O.C., Compositional attributes of the calyces of roselle (Hibiscus sabdariffa), J. Food Technol. Afr. 6 (4) (2001) 133-134.

[8] Bloomfield N., Preparation of sorrel ketchup from the red calyces of the roselle fruit (H. sabdariffa), Res. Proj. Rep., Food Sci. Technol. Unit, Fac. Eng., Univ. West Indies, St. Augustine, Trinidad Tobago, 1976.

[9] Dafallah A.A., al-Mustafa Z., Investigation of the anti-inflammatory activity of Acacia nilotica and Hibiscus sabdariffa, Am. J. Chin. Med. 24 (1) (1996) 263-269. 
[10] Maiga A., Diallo D., Bye R., Paulsen B.S., Determination of some toxic and essential metal ions in medicinal and edible plants from Mali, J. Agric. Food Chem. 53 (2005) 2316-2321.

[11] Lukaski H. C., Vitamin and mineral status: effects on physical performance, Nutr. 20 (2004) 632-644.

[12] Haider S., Naithani V., Barthawal J., Kakkar P., Heavy metal content in some therapeutically important medicinal plants, Bull. Environ. Contam. Toxicol. 72 (2004) 119-127.

[13] Bahemuka T.E., Mubofu E.B., Heavy metals in edible green vegetables grown along the sites of the Sinza and Msimbazi rivers in DarEs-Salaam, Tanzania, Food Chem. 66 (1999) 63-66.

[14] Tsai P.J., Studies on the color deterioration of roselles during post harvest handling, drying and storage, Natl. Chung Hsing Univ., Ph. D diss., Taiwan, 1995.

[15] Chen S.H., Huang T.C., Ho C.T., Tsai P.J., Extraction, analysis, and study on the volatiles in roselle tea, J. Agric. Food Chem. 46 (1998) 1101-1105.

[16] Forsyth W.G.C., Simmonds N.W., A survey of anthocyanins of some tropical plants, Proc. R. Soc. 142 (1954) 549-553.

[17] El-Adawy T.A., Khalil A.H., Characteristics of roselle seeds as a new source of protein and lipid, J. Agric. Food Chem 42 (1994) 18961900.

[18] Al-Wandawi H., Al-Shaikly K., Abdulrahman M., Roselle seeds: a new protein source, J. Agric. Food Chem. 32 (1984) 510-512.

[19] Mazza G., Miniati E., Anthocyanin in fruits, vegetables and grains, CRC Press, Boca Raton, FL, USA, 2000

[20] Du C.T., Francis F.J., Anthocyanins of roselle (Hibiscus sabdariffa L.), J. Food Sci. 38 (1973) 810-812.

[21] Pouget M.P., Vennat B., Lejeune B., Pourrat A., Extraction analysis and study of the stability of Hibiscus anthocyanins, Lebensm.Wiss. Technol, 23 (1990) 103-105.

[22] Bridle P., Timberlake C.F., Anthocyanins as natural food colours selected aspects, Food Chem. 58 (1997) 103-109.

[23] Degenhardt A., Knapp H., Winterhalter P., Separation and purification of anthocyanins by high-speed countercurrent chromatography and screening for antioxidant activity, J. Agric. Food Chem. 48 (2000) 338-343.
[24] Kahkonen M.P., Heinonen M.,. Antioxidant activity of anthocyanins and their aglycons, J. Agric. Food Chem. 51 (2003) 628-633.

[25] Lazze M.C., Pizzala R., Savio M., Stivala L.A., Prosperi E., Bianchi L., Anthocyanins protect against DNA damage induced by tert-butyl-hydroperoxide in rat smooth muscle and hepatoma cells, Mutat. Res. 535 (2003) 103-115.

[26] Palé É., Kouda-Bonafos M., Nacro M., Caractérisation et mesure des activités antiradicalaires d'anthocyanes de plantes du Burkina Faso, C. R. Chimie 7 (2004) 973980.

[27] Francis F.J., Colour analysis, in: Nielsen N.N., Food Analysis, Aspen Publ., Gaithersburg, MD, USA,1990, pp. 599-612.

[28] Wang C.J., Wang J.M., Lin W.L., Chu C.Y., Chou F.P., Tseng T.H., Protective effect of Hibiscus anthocyanins against tert-butyl hydroperoxide-induced hepatic toxicity in rats, Food Chem. Toxicol. 38 (2000) 411416.

[29] Herrera-Arellano A., Flores-Romero S., Chavez-Sotoc M., Tortoriello J., Effectiveness and tolerability of a standardized extract from Hibiscus sabdariffa in patients with mild to moderate hypertension: a controlled and randomized clinical trial, Phytomed. 11 (2004) 375-382.

[30] Dickel M.L., Rates S.M.K., Ritter M.R., Plants popularly used for loosing weight purposes in Porto Alegre, South Brazil, J. Ethnopharmacol. 109 (2007) 60-71.

[31] Tsai P.J., Mclntosh J., Pearce P., Camden B., Jordan B.R., Anthocyanin and antioxidant capacity in roselle (Hibiscus sabdariffa L.) extract, Food Res. Int. 35 (2002) 351-356.

[32] Chen H.H., Tsai P.J., Chen S.H., Su Y.M., Chung C.C., Huang T.C., Grey relational analysis of dried roselle (Hibiscus sabdariffa L.), J. Food Process. Preserv. 29 (2005) 228245

[33] Esselen W.B., Sammy G.M., Applications for roselle as a red food colorant, Food Prod. Dev. 9 (1975) 34-40.

[34] Tsai P.J., Ou A.S.M., Colour degradation of dried roselle during storage, Food Sci. 23 (1996) 629-640.

[35] Sarni-Manchad P., Cheynier V., Les polyphénols en agroalimentaire, Éd Tec \& Doc., Coll. Sci. \& Techn. Agroaliment., Lavoisier, Paris, 2006, 398 p. 
[36] Omobuwajo T.O., Sanni L.A., Balami Y.A., Physical properties of sorrel (Hibiscus sabdariffa) seeds, J. Food Eng. 45 (2000) 37-41.

[37] Oje K., Ugbor E.C., Some physical properties of oil bean seed, J. Agric. Eng. Res. 50 (1991) 305-313.

[38] Lazos E.S., Tsaknis J., Lalas S., Characteristics and composition of tomato seed oil, Grasas Aceites 49 (1998) 440-445.

[39] Diop A.G., Sakho M., Dornier M., Cisse M., Reynes M., Le baobab africain (Adansonia digitata L.) : principales caractéristiques et utilisations, Fruits 61 (1) (2005) 55-69.

[40] Glew R.H., Vanderjagt D.J., Lockett C., Grivetti L.E., Smith G.C., Pastuszyn A., Millson M., Amino acid, fatty acid, and mineral composition of 24 indigenous plants of Burkina Faso, J. Food Compos. Anal. 10 (1997) 205-217.

[41] Mohamed R., Fernandez J., Pineda M., Aguilar M., Roselle (Hibiscus sabdariffa) seed oil is a rich source of $\gamma$-tocopherol, J. Food Sci. 72 (3) (2007) 207-211.

[42] Abu-Tarboush H.M., Ahmed, S.B., Al Khatani H.A., Some nutritional and functional properties of karkade (Hibiscus sabdariffa) seed products, Cereal Chem. 74 (1997) 352-355.

[43] Swern D., Bailey's industrial oil and fat products, John Wiley \& Sons, Vols. 1 \& 2, N. Y., USA, 1979.

[44] Lewis M.J., Physical properties of foods and food processing systems, Ellis Horwood, London, UK, 1987.

[45] Uceda M., Hermoso M., La calidad de aceite de oliva, in: Barranco D., FernandezEscobar R., Rallo L. (Eds.), El cultivo del olivo, Ed. Mundi Prensa, Madrid, Spain, 2001.

[46] Oomah B.D., Ladet S., Godfrey D.V., Liang J., Girard B., Characteristics of raspberry (Rubus idaeus L.) seed oil, Food Chem. 69 (2000) 187-193.

[47] Jirovetz L., Jaeger W., Remberg G., Espinosa-Gonzalez J., Morales R., Woidich A., Nikiforov A., Analysis of the volatiles in the seed oil of Hibiscus sabdariffa (Malvaceae) by means of GC-MS and GC-FTIR, J. Agric. Food Chem. 40 (1992) 1186-1187.

[48] Leung W., Busson F., Jardin C., Food composition table for use in Africa, FAO, Rome, Italy, 1968, $306 \mathrm{p}$.

[49] Diouf M., Lô C., Gueye M., Mbengue N.B., Sélection participative de nouveaux cultivars de quatre (4) espèces de légumes feuilles
(Hibiscus sabdariffa L., Amaranthus L. spp., Vigna unguiculata (L.) Walp et Moringa oleifera Lam) au Sénégal, Afr. J. Food Agric. Nutr. Dev. 7 (3) (2007).

[50] Attere F., Note introductive de l'atelier sur les légumes feuilles traditionnels, in: Chweya J.A, Eyzaguire P. (Eds.), Biodiversity of traditional leafy vegetables in Africa, Int. Plant Genet. Res. Inst. (IPGRI), Rome, Italie, 1999.

[51] Kahane R., Temple L., Brat P., De Bon H., Les légumes feuilles dans les pays tropicaux : diversité, richesse économique et valeur santé dans un contexte très fragile, in : Les légumes : un patrimoine à transmettre et à valoriser, colloq. AFCEV-BRG-INH, 7-9 Sept. 2005, Angers, France.

[52] Mounigan P., Badrie N., Physicochemical and sensory quality of wines from red sorrel/ roselle (Hibiscus sabdariffa L.) calyces: effects of pretreatments of pectolase and temperature/time, Int. J. Food Sci. Technol. 42 (2007) 469-475.

[53] Ojokoh A.O., Adetuyi F.C., Akinyosoye F.A., Oyetayo V.O., Fermentation studies on roselle (Hibiscus sabdariffa) calyces neutralised with trona, J. Food Technol. Afr. 7 (3) (2002) 75-78.

[54] Ouedraogo J., Vinaigre d'oseille, SYFIA 99, 1997.

[55] Odunfa S.A., Microorganisms associated with fermentation of African locust bean (Parkia filicoidea) during 'iru' preparation, J. Plant Foods 3 (1981) 245-250.

[56] Dashak D.A., Dawang M.L., Lucas N.B., An assessment of the proximate chemical composition of locally produced spices known as dadawa basso and dadawa kalwa from three markets in Plateau State of Nigeria, Food Chem. 75 (2001) 231-235.

[57] Beaumont M., Flavouring composition prepared by fermentation with Bacillus spp., Int . J. Food Microbiol. 75 (2002) 189-196.

[58] Yagoub A.A.., Mohamed E.B., Ahmed A.H.R., El Tinay A.E., Study on furundu, a traditional Sudanese fermented roselle (Hibiscus sabdariffa L.) seed: effect on in vitro protein digestibility, chemical composition, and functional properties of the total proteins, J. Agric. Food Chem. 52 (2004) 6143-6150.

[59] Diawara B., Sawadogo L., Amoa-Awua W.K.A., Jakobsen M., Quality system for the production of soumbala, The HACCP System, Waitro, Dan. Technol. Inst., Taastrup, Dan., 1998. 
[60] N'Dir B., Hbid C., Cornelius C., Roblain D., Jacques P., Vanhentenryck F., Diop M., Thonart P., Propriétés antifongiques de la microflore sporulée du nététu, Cah. Agric. 3 (1994) 23-30.

[61] Wang J., Fung Y.C., Alkaline-fermented foods. A review with emphasis on Pidan fermentation, Crit. Rev. Microbiol. 22 (2) (1996) 101-138.

[62] Babatunde F.E., Intercrop productivity of roselle in Nigeria, Afr. Crop Sci. J. 11 (2003) 1-6.

[63] Diouf M., Diop M., Lô C., Drame K.A., Sene E., Ba C.O., Gueye M., Faye B., Prospection de légumes feuilles traditionnels de type africain au Sénégal, in: Chweya J.A, Eyzaguire P. (Eds.), Biodiversity of traditional leafy vegetables in Africa, Int. Plant Genet. Res. Inst. (IPGRI), Rome, Italy, 1999, pp. 111-150.

[64] Ajay M., Achike F.I., Mustafa A.M., Mustafa M.R., Direct effects of quercetin on impaired reactivity of spontaneously hypertensive rat aortae. Comparative study with ascorbic acid, Clin. Exp. Pharmacol. Physiol. 33 (2006) 345-350.

[65] Ajay M., Chai H.J., Mustafa A.M., Gilani A.H Mustafa M.R., Mechanisms of the antihypertensive effect of Hibiscus sabdariffa L. calyces, J. Ethnopharmacol. 109 (2007) 388-393.

[66] Kirdpon S., Nakorn S.N., Kirdpon W. Changes in urinary chemical composition in healthy volunteers after consuming roselle (Hibiscus sabdariffa L.) juice, J. Med. Assoc. Thail. 77 (1994) 314-321.

[67] Haji F.M., Haji T.A., The effect of sour tea (Hibiscus sabdariffa) on essential hypertension, J. Ethnopharmacol. 65 (1999) 231-236.

[68] Odigie I.P., Ettarh R.R., Adigun S.A., Chronic administration of aqueous extract of Hibiscus sabdariffa attenuates hypertension and reverses cardiac hypertrophy in $2 \mathrm{~K}-1 \mathrm{C}$ hypertensive rats, J. Ethnopharmacol. 86 (2003) 181-185.

[69] Hirunpanich V., Utaipat A., Morales N.P., Bunyapraphatsara N., Sato H., Herunsale A., Suthisisang C., Hypocholesterolemic and antioxidant effects of aqueous extracts from the dried calyx of Hibiscus sabdariffa L. in hypercholesterolemic rats, J. Ethnopharmacol. 103 (2006) 252-260.

[70] Onyenekwe P.C., Ajani E.O., Ameh D.A., Gamaniel K.S., Antihypertensive effect of roselle (Hibiscus sabdariffa) calyx infusion in spontaneously hypertensive rats and a comparison of its toxicity with that in Wistar rats, Cell Biochem. Funct. 17 (1999) 199-206.

[71] Suboh S.M., Bilto Y.Y., Aburjai T.A., Protective effects of selected medicinal plants against protein degradation, lipid peroxidation and deformability loss of oxidatively stressed human erythrocytes, Phytother. Res. 18 (2004) 280-284.

[72] Ali B.H., Mousa H.M., El-Mougy S., The effect of a water extract and anthocyanins of Hibiscus sabdariffa L. on paracetamolinduced hepatoxicity in rats, Phytother. Res. 17 (1) (2003) 56-59.

[73] Christiana K.R., Nairb M.G., Jackson J.C., Antioxidant and cyclooxygenase inhibitory activity of sorrel (Hibiscus sabdariffa), J. Food Compos. Anal. 19 (2006) 778-783.

[74] Chen C.C., Hsu J.D., Wang S.F., Chiang H.C., Yang M.Y., Kao E.S., Ho Y.C., Wang C.J.,. Hibiscus sabdariffa extract inhibits the development of atherosclerosis in cholesterol-fed rabbits, J. Agric. Food Chem. 51 (2003) 5472-5477.

[75] Prenesti E., Berto S., Daniele P.G., Toso S., Antioxidant power quantification of decoction and cold infusions of Hibiscus sabdariffa flowers, Food Chem. 100 (2007) 433-438.

[76] Lin W.L., Hsieh Y.J., Chou F.P., Wang, C.J., Cheng M.T., Tseng T.H., Hibiscus protocatechuic acid inhibits lipopolysaccharideinduced rat hepatic damage, Arch. Toxicol. 77 (2003) 42-47.

[77] Wang C.J., Wang J.M., Lin W.L., Chu C.Y., Chou F.P., Tseng T.H., Protective effect of Hibiscus anthocyanins against tert-butyl hydroperoxide-induced hepatic toxicity in rats, Food Chem. Toxicol. 38 (2000) 411416.

[78] Amin A., Hamza A.A., Hepatoprotective effects of Hibiscus, Rosmarinus and Salvia on azathioprine-induced toxicity in rats, Life Sci. 77 (2005) 266-278.

[79] Falade O.S., Otemuyiwa I.O., Oladipo A., Oyedapoc O.O., Akinpelu B.A., Adewusi S.R.A., The chemical composition and membrane stability activity of some herbs used in local therapy for anaemia, J. Ethnopharmacol. 102 (2005) 15-22.

[80] Mounnissamy V.M., Gunasegaran R., Gopal V., Saraswathy A., Diuretic activity of gossypetin isolated from Hibiscus sabdariffa in rats, Hamdard Medicus 45 (2) (2002) 68-70. 
[81] Tseng T.H., Hsu J.D., Lo M.H., Chu C.Y., Chou F.P., Huang C.L., Wang C.J., Inhibitory effect of Hibiscus protocatechuic acid on tumor promotion in mouse skin, Cancer Lett. 126 (1998) 199-207.

[82] Lin L.T., Liu L.T., Chiang L.C., Lin C.C., In vitro antihepatoma activity of fifteen natural medicines from Canada, J. Sci. Food Agric. 16 (2002) 440-444.

[83] Chen C.C., Chou F.P., Ho Y.C., Lin W.L., Kao E.S., Huang A.C., Wang C.J., Inhibitory effects of Hibiscus sabdariffa L. extract on low-density lipoprotein oxidation and anti-hyperlipidemia in fructose-fed and cholesterol-fed rats, J. Sci. Food Agric. 84 (2004) 1989-1996.

[84] Chang Y.C., Huang K.X., Huang A.C., Ho Y.C., Wang C.J., Hibiscus anthocyanins-rich extract inhibited LDL oxidation and oxLDL- mediated macrophages apoptosis, Food Chem. Toxicol. 44 (2006) 1015-1023.

[85] Garcia V.M.N., Rojas G., Zepeda L.G., Aviles M., Fuentes M., Herrera A., Enrique Jiménez E., Antifungal and antibacterial activity of four selected Mexican medicinal plants, Pharm. Biol. 44 (4) (2006) 297-300.

[86] Liu J.Y., Chen C.C., Wang W.H., Hsu J.D., Yang M.Y., Wang C.J., The protective effects of Hibiscus sabdariffa extract on $\mathrm{CCl}_{4}$ induced liver fibrosis in rats, Food Chem. Toxicol. 44 (2006) 336-343.

[87] Akindahunsi A.A., Olaleye M.T., Toxicological investigation of aqueous-methanolic extract of the calyces of Hibiscus sabdariffa L., J. Ethnopharmacol. 89 (2003) 161-164.

[88] Kohen J.L., Downing A.J., Aboriginal use of plants on the western Cumberland Plain Sydney Basin, Naturalist 1 (1992) 1-6.

\section{El bissap (Hibiscus sabdariffa L.): composición y principales utilizaciones.}

Resumen - Introduction. Hibiscus sabdariffa L. es una planta herbácea, que se encuentra en gran parte en áreas tropicales y subtropicales de ambos hemisferios. H. sabdariffa es utilizado por sus fibras, y principalmente por sus cálices que son de tres tipos: verde, rojo y rojo oscuro. Composición. Los cálices tipo rojo son los más utilizados y se caracterizan por su alta concentración de antocianinas, que puede llegar a $1,5 \mathrm{~g} \cdot \mathrm{kg}^{-1}$. El delphinidine 3-sambubioside y cyanidine 3-sambubioside antocianinas son la mayoría, respectivamente con (71 y el 29) \% del total de antocianinas. Los ácidos orgánicos, minerales y aminoácidos están presentes en los cálices, hojas y semillas de $H$. sabdariffa a los niveles varían dependiendo de la variedad y zona geográfica. Usos. El uso de diferentes partes del $H$. sabdariffa son muchas y variadas, tanto en los alimentos que en la medicina tradicional. Los cálices, debido a su alta concentración de ácido, vitamina $\mathrm{C}$ y especialmente en antocianinas son parte de la planta más utilizada. Ellos son utilizados principalmente para la producción de bebidas refrescante y tónico sin alcohol. Las semillas de $H$. sabdariffa por su riqueza en proteínas (26\%), grasa (20\%) y azúcares totales (40\%) son ampliamente utilizados en la dieta de muchos países africanos. Las hojas son una buena fuente de nutrientes y se utilizan en África en la preparación de salsas.

Senegal / Hibiscus sabdariffa / cáliz / hojas / semilla / antocianinas / composición aproximada / usos 